\title{
Belphégor
}

Littérature populaire et culture médiatique

17 | 2019

Mutations des légitimités dans les productions culturelles contemporaines

\section{Les fans, ces publics si spécifiques. Définition et méthodologie pour le chercheur}

\section{Mélanie Bourdaa}

\section{(2) OpenEdition \\ Journals}

Electronic version

URL: https://journals.openedition.org/belphegor/1701

DOI: 10.4000/belphegor.1701

ISSN: 1499-7185

Publisher

LPCM

Electronic reference

Mélanie Bourdaa, "LES faNS, CES PUblics SI SPÉcIfiques. DÉfinITION ET MÉTHOdOLOgIE POUR LE CHERCHEUR", Belphégor [Online], 17 | 2019, Online since 18 April 2019, connection on 25 August 2021. URL: http://journals.openedition.org/belphegor/1701 ; DOI: https://doi.org/10.4000/belphegor. 1701

This text was automatically generated on 25 August 2021.

\section{(c)}

Belphégor est mis à disposition selon les termes de la Licence Creative Commons Attribution - Pas d'Utilisation Commerciale - Pas de Modification 4.0 International. 


\title{
Les fans, ces publics si spécifiques. Définition et méthodologie pour le chercheur
}

\author{
Mélanie Bourdaa
}

1 Les fans font l'objet de discours, surtout médiatiques, qui tendent à dresser des portraits stéréotypés, décrivant des fanboys obsessionnels et des fangirls hystériques ${ }^{1}$. Pourtant, si l'on analyse ces publics spécifiques de la pop culture et des industries culturelles, nous pouvons rapidement constater que ces stéréotypes ne tiennent pas face aux activités de réflexion et de création que mettent en place les fans dans des communautés de pratiques, créant ainsi des formes de résistance et une sous-culture propre.

2 Cet article se propose, à travers une revue de la littérature critique, de revenir à la fois sur les définitions du fan et de la fan culture et de comprendre comment les chercheurs peuvent appréhender ces publics, à travers des méthodologies spécifiques adaptées au contexte et aux cultures fans. Nous verrons ainsi comment l'identité du chercheur peut se lier avec celle du fan qu'il est, et comment son statut de fan peut irriguer ses pratiques de chercheurs et lui donner une légitimité pour entreprendre des recherches dans les communautés de fans.

\section{Comment définir les fans?}

« Tout le monde sait ce qu'est un fan. C'est quelqu'un qui est obsédé par une star en particulier, une célébrité, un film, un programme télévisé, un groupe ; quelqu'un qui peut offrir des informations sur son fandom, peut citer ses lignes de dialogue favorites ou les paroles de chanson, les vers ou chapitre qu'il préfère. Les fans sont très éloquents. Les fans interprètent les textes médiatiques de manières très variées, parfois surprenantes mais toujours intéressantes. Et les fans participent à des activités communes, ils ne sont pas des spectateurs ou lecteurs isolés. Donc, comment les communautés de fans et les cultes médiatiques ont-ils été définis par les chercheurs universitaires? Encore aujourd'hui, il est difficile de définir les communautés de fans à cause de (ou peut-être grâce à) la banalité du terme $»^{2}$. 
Il est extrêmement complexe de définir les fans car le terme recouvre un grand nombre de pratiques et une variété de définitions qui sont l'objet de tensions dans les milieux académiques et dans les croyances populaires, mais également de pratiques. En France, le terme fan conserve pour beaucoup sa tonalité péjorative et reste associé avec le culte religieux. De fait les fans ont longtemps été perçus comme des publics aliénés, dépendant des industries culturelles et créatives. En 1979, Bourdieu dans La Distinction ${ }^{3}$ décrit le fan comme dominé et n'ayant aucune distance par rapport à sa passion. Gabriel Segré, dans son analyse des fans de célébrités et de vedettes, émaille son ouvrage de termes rappelant la marginalisation des fans et leur dévotion quasi religieuse : « ils (les fans) font parfois de cette célébration posthume une quasi-religion, avec ses fidèles, ses rites, ses cérémonies, ses croyances, ses lieux et ses objets sacrés, ses églises $»^{4}$. Aujourd'hui, un écueil supplémentaire consiste en ce que tout le monde se définit comme fan de quelque chose, sans pour autant comprendre les enjeux d'identité, de créativité et de partage que porte en elle la notion. Lorsque des personnes se définissent comme des fans, c'est pour signaler qu'elles regardent régulièrement une série télévisée ou un film par exemple. Lors d'une recherche sur les fans de Game of Thrones menée avec Javier Lozano Delmar, nous avions demandé aux publics français et espagnols pourquoi ils se considéraient comme des fans de la série et la plupart d'entre eux avaient mis en avant un aspect quantitatif et technique pour se définir: le visionnage et le revisionnage systématique des épisodes. Un fan nous avait par exemple répondu: «parce que je ne me lasse pas de regarder les épisodes » et un autre "parce que je suis la série avec assiduité $»^{5}$. Le fait de regarder les épisodes souvent plus d'une fois garantit à ces téléspectateurs français et espagnols un statut de fan, qu'ils n'arrivent pas eux-mêmes à bien définir et qui repose sur un aspect quantitatif plus que sur un aspect qualitatif. C'est en effet presque davantage l'intensité du visionnage qui est valorisée que les pratiques et activités que décrivait Jenkins dans son analyse des Trekkies ${ }^{6}$. Ce décalage qui existe dans les perceptions est entre autres un problème culturel. En effet, aux États-Unis, les publics ont moins de réticence à s'auto-proclamer fans, et intègrent dans cette définition de soi des activités de création, de partage, d'engagement civique. Il y a eu bien entendu une évolution assez flagrante. Par exemple, Paul Booth explique ce changement dans ses cours sur les fans en soulignant qu'aujourd'hui ceux qui s'y inscrivent connaissent les pratiques de fans et participent souvent aux activités d'une ou de plusieurs communautés de fans. Pour ma part, lorsque je pose cette question dans mon propre cours, je ressens toujours une gêne de la part des étudiants, dont très peu avouent être fans.

4 Les préjugés et les stéréotypes jouent un rôle déterminant dans ces perceptions puisqu'ils persistent encore, notamment dans les représentations véhiculées par les médias sur les fans. Paul Booth et Lucy Bennett ${ }^{7}$, dans un ouvrage collectif qu'ils ont dirigé, cherchent d'ailleurs à comprendre "jusqu'où les stéréotypes perpétuent les mythes autour des communautés de fans et de la relation affective qu'ils entretiennent avec les textes médiatiques» (p. 6) dans les médias, tous supports confondus (documentaires, presse, séries TV, films). Pour cela, ils se réfèrent à des études qui listent les stéréotypes associés aux fans dans les médias. Par exemple, Jensen en 1992 notait que les fans étaient souvent qualifiés de " consommateurs sans cerveau » et de "désaxés asociaux» qui accordent trop d'importance au texte culturel et qui sont incapables de séparer la réalité de la fiction (p. 14). Jenkins notait lui-même dans son ouvrage fondateur que les qualificatifs qui revenaient le plus souvent pour définir les 
fans étaient « marginaux » et « hors de contrôle ». Plusieurs chapitres prennent comme exemple le sketch de William Shatner intitulé « Get a life » diffusé lors de l'émission de talk show américaine Saturday Night Life dans lequel il fustige les fans de Star Trek (il joue le personnage du Captain Kirk, capitaine du vaisseau Enterprise) et les dépeint comme des gens obsessionnels qui n'ont pas de vie en dehors de leur passion pour la série. Ce sketch représente « la quintessence des stéréotypes » sur les fans qui persistent encore aujourd'hui dans les représentations médiatiques. Souvent les fans masculins sont représentés comme obsessionnels et asociaux (dans le style des Otaku japonais) cherchant les moindres détails de l'univers narratif et collectionnant les moindres objets liés à cet univers alors que les jeunes filles fans sont présentées comme des personnes hystériques qui ne savent pas se contrôler, en particulier lorsqu'apparaissent leurs idoles et célébrités favorites. Tout le monde peut se souvenir de reportages sur les fans des Beatles ou les fans de groupes de musique comme Tokio Hotel ou One Direction, qui éclatent en sanglot ou s'évanouissent lors des concerts ou interviews. Ces stéréotypes continuent de perdurer et sont renforcés par les reportages, documentaires ou séries télévisées qui marginalisent la plupart du temps les fans dans leurs pratiques, notamment lorsque sont abordés le cosplay ou l'écriture de fan fictions. Seule la facette négative est montrée, pour le sensationnalisme et la dramatisation, au détriment des activités que les fans mettent en place dans leur communauté et en occultant la réflexivité sur une passion qu'ils transforment en action et en création.

5 Pour aller au-delà de ces stéréotypes et bien comprendre ce que sont les fans aujourd'hui, il est important ici de clarifier notre définition du fan. Pour nous, le fan n'est pas seulement un "suiveur", un collectionneur ou un enthousiaste, pour reprendre les catégories de Longhurst et Abercrombie ${ }^{8}$, il ne placarde pas seulement ses murs de posters comme l'avait souligné Fiske ${ }^{9}$, mais il est aussi un producteur de contenus et de signification. En ce sens, nous nous rapprochons de la définition pionnière de Jenkins qui voit dans les fans des «consommateurs qui produisent, des lecteurs qui écrivent, des spectateurs qui participent $\aleph^{10}$. Malgré cette définition d'un fan actif, producteur participant et engagé, Jenkins ne sous-estime pas l'importance d'un public non producteur qui est toutefois engagé dans une réception systématique et répétée, correspondant aux profils des fans espagnols et français évoqués plus haut. Francesca Coppa rappelle ce positionnement de Jenkins, que nous pourrions facilement oublier tant l'auteur a surtout mis en avant les fans producteurs et acteurs de leur réception :

«Jenkins comprend qu'il y a des fans qui simplement vont regarder un programme religieusement, en parler, et pourtant ne s'engager dans aucune activité ou pratique de fans ; une telle manière d'agir est celle des fans de Dallas décrits par Ien Ang mais aussi de la plupart des téléspectateurs aujourd'hui (et à peu près tous les gens possédant une télévision dans les dernières semaines de Breaking Bad)» (Gilligan, 2008). Jenkins a clarifié ses propos dans Science Fiction Audiences (1995) co-écrit avec Tulloch :

"'Ce livre va par conséquent proposer une distinction entre les fans (publics actifs à l'intérieur d'une communauté en tant qu'institution culturelle, sociale et interprétative), et les suiveurs (publics qui regardent et apprécient des programmes de science-fiction mais qui n'affirment aucune identité sociale plus large sur la base de cette consommation) (p. 23)' $»^{11}$.

6 Lors du tournant ethnographique des Cultural Studies, et dans les premières études de réception qui s'apparentent aux études de fans actuelles, Ien $\mathrm{Ang}^{12}$, dans son étude sur 
la série américaine Dallas par un public non-américain, avait surtout analysé ce public spécifique en le qualifiant de Dallas lovers, ce que nous pourrions traduire par les « amoureux » de Dallas. Cependant, il s'est avéré que ce public regardait simplement la série sans s'engager dans des activités périphériques et sans produire de contenus originaux partagés ensuite dans une communauté.

Pour Sarah M. Corse et Jaime Hartless,

"les fans, et particulièrement dans la communauté de science-fiction, peuvent dépenser des sommes considérables, du temps et de l'énergie pour leurs identités culturelles, se rendre au Comic Con de San Diego, fabriquer des costumes, écrire des fan fiction ou de la musique, et participer à des communautés en ligne pour partager leurs intérêts avec d'autres membres qui sont engagés de la même façon $»^{13}$.

8 Les deux auteurs notent d'emblée les notions d'engagement à la fois émotionnel et matériel, en termes d'argent et de temps, qui va caractériser le lien entre les fans euxmêmes au sein de la communauté et entre les fans et l'œuvre ou les œuvres culturelles et médiatiques qui les passionnent. Ce sont également ces points saillants que met en avant Paul Booth dans son ouvrage Digital Fandom, tout en soulignant que c'est souvent la définition commune du fan: " dans le langage commun, le fan est une personne qui investit du temps et de l'énergie pour penser et interagir avec un texte médiatique ${ }^{14}$. Ces définitions pourraient facilement limiter le fan à un consommateur, prisonnier du système capitaliste que lui imposent les médias contemporains. Mark Duffet nous rappelle d'ailleurs que «les consommateurs sont des récepteurs aliénés qui se complaisent dans la chaîne de circulation économique en dépensant de l'argent en échange de services et de biens $»^{15}$. Or il convient de ne pas considérer les fans comme de simples consommateurs passifs de produits culturels, puisque leurs propres productions vont entrainer des questionnements sur l'identité sexuelle, le racisme, le genre, le colonialisme par exemple et que les communautés agissent comme des agents d'empowerment ou parfois de désempowerment. Le fan et les communautés de fans ne sont donc pas des entités passives, loin de là, et nous voudrions souligner que les fans produisent, écrivent, montent des vidéos, créent, s'engagent dans le politique, débattent. Patrick Flichy met également ces points en avant lorsqu'il écrit que « les fans prolongent leur réception par une activité créatrice et hétérodoxe qui se manifeste soit par des ajouts à la fiction, soit par un collage de différents éléments $»^{16}$ (p. 32). C'est ce que l'on pourrait qualifier à la suite de Laurence Allard ${ }^{17}$ de "culture du remix » - art ou esthétique à part entière. Les fans expérimentent en réalité une réception sans fin, puisque le moment de leur réception d'une œuvre va au-delà de la lecture ou du visionnage, mais s'étend lors de discussions, d'activités, de partages, d'engagements civiques.

9 En résumé, être fan, pour nous, c'est attester des caractéristiques suivantes :

10 - Appartenir à une communauté de pairs, à un groupe social dans lequel le fan va inscrire sa passion et dont il va devenir membre. Cette communauté présente une grande organisation, possède ses codes mais également ses hiérarchies, et ses sanctions et tensions.

11 - Allonger, étirer et déployer le moment de la réception pour que celle-ci ne se termine pas et constitue une boucle infinie.

12 - Etre un public expert, puisque le fan connait l'œuvre suffisamment pour s'adonner à un braconnage culturel, source de sa créativité. 
13 - Etre un producteur de sens et de contenus. Le fan réinterprète les œuvres, les décortique, les analyse, propose des remix et des collages, des mélanges de genres et d'œuvres. Il crée des productions qui vont de l'écriture de fan fictions au montage vidéo, ou encore au dessin de fan art.

14 - S'engager socialement, culturellement et politiquement pour déployer son identité personnelle et collective dans la sphère publique.

15 Le degré d'engagement du fan dans la communauté varie en fonction de ses intérêts et des autres communautés (fandoms) dans lesquelles il est engagé. En effet, comme le rappelle Nancy Baym ${ }^{18}$, les publics migrent désormais d'un fandom à un autre, et disséminent leurs passions au gré de leurs migrations. Les paliers dans l'investissement du fan nous permettent d'établir des catégories lors de notre travail d'enquête. Par exemple, Christian Le Bart et Jean-Charles Ambroise, lors de leur étude sur les fans des Beatles, avaient proposé de classer les fans en six catégories suivant leur engagement: le créateur, l'érudit, la groupie, l'esthète, le collectionneur, l'imitateur ${ }^{19}$. Nos catégories seront bien entendu différentes puisque nous analyserons les fans de productions audiovisuelles.

\section{Comment étudier les fans?}

16 Les méthodologies appliquées aux études de fans ont souvent été questionnées ou critiquées. Dans un article de 2014, Adrienne Evans et Mafalda Stasi ${ }^{20}$ s'interrogent sur le manque d'articles traitant des questions méthodologiques liées aux études de fans, si l'on excepte ceux sur la position de l'aca-fan (academic-fan) chère à Jenkins, et s'étonnent du grand nombre de recherches sur les fans qui n'exposent pas explicitement leur méthodologie. Il est important de noter que les fans studies, les études de fans, se sont construites au croisement de plusieurs disciplines, ce qui peut expliquer ce manque de méthode claire : anthropologie, sociologie, littérature anglosaxonne, études cinématographiques, études médiatiques, cultural studies. Chacune de ces disciplines possède ses propres méthodologies quantitatives et qualitatives qu'il faut ensuite réinvestir et souvent remodeler pour analyser les communautés de fans. En France, les études de fans sont présentes en sociologie des publics et elles constituent un champ de recherche émergent en Sciences de l'Information et de la Communication $^{21}$. Mais se pose tout de même la question de la méthodologie à employer.

17 Dès les études pionnières des années 90 , deux types de méthodes se confrontaient : une analyse de l'intérieur de la communauté (Jenkins) et une analyse en surplomb de la communauté (Bacon-Smith). Les fans qui étaient l'objet des études voyaient dans la position du chercheur à l'intérieur de la communauté la garantie d'une bienveillance vis-à-vis de leur pratique, ce qui conférait au chercheur une certaine légitimité. "Jenkins travaillait avec les fans pour trouver leurs propres voix, et Bacon-Smith regardait les fans dans une perspective académique. Les études de fans aujourd'hui devraient tirer leçon du passé, donner une place aux voix des fans en tant que partie intégrante de la recherche, et produire des recherches additionnelles à destination des publics fans $»^{22}$. Ici, s'oppose une posture "à l'intérieur de" et une posture "à l'extérieur de », ou de chercheur participant versus chercheur non-participant. 
18 La position adoptée par Jenkins a été qualifiée d'aca-fan : un chercheur qui travaille sur une communauté qu'il a de fait, par son statut de fan, déjà intégrée avant le début de sa recherche. Matt Hills propose la position du fan-scholar, qui à l'inverse est un fan qui décide d'entreprendre une recherche sur sa communauté. L'avantage de ses deux positionnements, qui avait déjà été souligné, est la connaissance quasi encyclopédique de la communauté étudiée. Il existe donc une double identité entre le chercheur et le fan, qui complique la définition de l'aca-fan. En effet, Matt Hills note à juste titre que de plus en plus de fans s'adonnent à des analyses de style académique lorsqu'ils créent des blogs, qu'ils participent à des forums de discussion, qu'ils écrivent des analyses rhétoriques pour interpréter les motivations de personnages, qu'ils convoquent les théories de la narratologie pour comprendre les intrigues. L'immersion participative (ou l'observation participante dans la communauté de fans), confère au chercheur un regard pertinent sur les pratiques des fans. Comme le rappelle Aurore Gallarino, «l'acafan produit donc une analyse co-construite avec ses sujets ${ }^{23}$. Paul Booth, quant à lui, utilise le terme de Fan-Scholar, "un fan utilisant des concepts et références universitaires pour produire un discours destiné à d'autres fans, dans une publication destinée aux fans, blog ou autre $\aleph^{24}$.

Bien entendu, les chercheurs ont dû s'adapter aux pratiques de fans et se déplacer également vers les communautés de fans essentiellement en ligne aujourd'hui. Désormais, les fans se rassemblent dans des communautés virtuelles, ce qui a pour conséquence de briser des frontières spatiales, géographiques et temporelles assez importantes pour créer des communautés mondiales de façon quasi instantanée. Cela impose au chercheur un repositionnement de ses méthodes et de sa démarche. En effet, "Depuis la sortie de Textual Poachers, Enterprising Women, et The Adoring Audience, seules quelques études ethnographiques off-line sur les fans ont été publiées (Hills, 2005 ; Lamerichs, 2013). Il existe une bonne raison à cela : en plus de la difficulté à trouver un échantillon assez large pour pouvoir généraliser, la facilité et l'accès d'Internet ont ouvert de grandes possibilités pour la recherche (Markham et Baym, 2008). De plus, les fans eux-mêmes se sont massivement rendus sur Internet, et les chercheurs les ont suivis (voir Jenkins, 2006 ; Stein, 2006) $»^{25}$. Les chercheurs doivent donc s'adapter aux pratiques des communautés de fans, qui sont considérées comme des "early adopters " des nouvelles technologies et qui développent rapidement des compétences technologiques. Comme le souligne Sam Ford en s'appuyant sur les recherches de Bird, "les activités de fans qui produisent leurs propres textes ont connu une attention extrême de la part des chercheurs dans le monde du Web 2.0 parce que les fans produisent une grande quantité de contenus en ligne qui peuvent être étudiés assez facilement, alors qu'un engagement moins intense est plus diffus et moins visible des chercheurs $»^{26}$ (Ford, 2014 : 63). Les fans sur Internet laissent des traces numériques multiples, mouvantes, créatives, ainsi que des textes, images, vidéos, discussions qui sont autant d'archives de leur passion mais également de leurs pratiques que le chercheur peut alors convoquer, saisir et analyser. Il peut se constituer une base de données immense sans cesse en augmentation, et avoir ainsi accès à un corpus quasi illimité de ressources faniques. Dès lors, si l'on cherche à mieux saisir les pratiques de réception des amateurs de séries télévisées lorsque l'on est chercheur, il devient nécessaire d'utiliser internet comme outil d'analyse. Les pratiques de réception des téléspectateurs et des fans ont évolué pour se déplacer sur internet, afin de créer des espaces d'échange, de partage, de création, et de rassemblement communautaire. Comme le rappelle fort justement Clément Combes, « la forte appétence pour les séries 
- que nous désignons par "sériephilie» - se donne notamment à voir de façon remarquable au travers des collectifs qui opèrent sur de multiples sites, blogs et forums en ligne " (Combes, $2011:$ 139). Pour Paul Booth, les pratiques de réception des fans se développent avant tout dans un environnement numérique. Sa définition de la communauté virtuelle atteste de cette nouvelle posture de réception: "par communauté, j'entends le groupe social d'individus partageant des intérêts communs, rassemblés par une adhésion ; l'organisation d'un groupe de fans qui à la fois aiment les œuvres médiatiques et qui créent des contenus additionnels autour de ce texte médiatique » (Booth, 2010: 22).Au vu de ces «nouvelles pratiques de réception », le chercheur est amené à analyser des pratiques virtuelles au sein de communautés, ou tout du moins des échanges ayant lieu sur la toile. La méthodologie la plus utilisée pour les études de fans est naturellement celle de l'ethnographie, l'analyse d'une communauté et de ses pratiques, codes, rites, qui permet d'insister à la fois sur la voix des fans et sur celle des chercheurs. L'ethnographie était la méthodologie utilisée dans les études des publics en Cultural Studies. En se basant sur les propositions de décodage de Stuart Hall ${ }^{27}$, les chercheurs ont mis en lumière et documenté, entre autres, les goûts et pratiques des publics familiaux ${ }^{28}$, des publics transculturels ${ }^{29}$ ou bien des publics féminins ${ }^{30}$. Cependant, ces méthodologies empruntées à l'ethnographie ont été fortement critiquées comme n'étant pas de la « vraie » ethnographie. Comme le précise Nightingale, l'analyse des lettres de fans et des entretiens auprès des fans, du point de vue de l'anthropologie, ne constituent pas une approche ethnographique des publics. Il est important de noter que malgré cette critique, les chercheurs en études de fans prônent l'usage de cette méthodologie allant même jusqu'à « s'engager dans une autoethnographie pour développer leur identité de fan et de chercheur $»^{31}$ (p. 125).

Depuis l'avènement des nouvelles technologies, on est passé d'une ethnographie à une cyberethnographie. Pour pratiquer une méthode de cyberethnographie, il faut employer une posture d'ethnographe, ce qui signifie qu'il faut intégrer la communauté que l'on va analyser. Ward ${ }^{32}$ rappelle que la cyberethnographie est une méthode utile qui permet une description pointue d'une communauté virtuelle. Cela se justifie par le fait que le chercheur, avec la cyberethnographie, « devient un participant de la société et fait des observations en tant que membre extérieur. Etant donné que les communautés virtuelles forment une culture et une société spécifiques, des données peuvent être collectées grâce à une méthode de cyberethnographie plutôt qu'avec n'importe quelle autre technique de recherche $»^{33}$ (p. 2). Atkuran exclut cependant l'appartenance à la communauté que le chercheur va étudier et postule une posture extérieure et de surplomb semblable à celle de Bacon-Smith, n'incluant donc pas dans sa posture méthodologique la position de l'aca-fan prônée par Jenkins.

21 L'analyse des communautés et des pratiques de fans requiert une posture de chercheur entre l'aca-fan, tel que défini par Henry Jenkins (c'est-à-dire un chercheur fan luimême de l'objet qu'il étudie), et l'observation des traces numériques laissées par les fans (lui octroyant une distance critique par rapport à son objet).

Malgré ces recommandations méthodologiques liées aux nouvelles pratiques de fans et à leur migration massive sur Internet, il ne faut pas tomber dans un technodéterminisme qui mettrait plus en valeur certaines communautés que d'autres ou qui marquerait des différences générationnelles prononcées. Il est nécessaire également de ne pas produire des hiérarchies culturelles à l'intérieur des fandoms ou d'un fandom à l'autre en mettant en avant certaines pratiques ou certaines formes d'engagement par 
rapport à d'autres. Paul Booth explique que les contenus médiatiques doivent être désormais appréhendés sous un angle nouveau : au vu des mutations des pratiques de réception, les chercheurs doivent désormais étudier la relation entre le texte, le médium et la technologie. Cela induit le changement de paradigme que Paul Booth décrit, entraînant de nouvelles études sur les médias. Nous retrouvons dans ces analyses tout ce qui fait la force de la communauté: un rassemblement, des compétences techniques, et un engagement intellectuel et émotionnel dans une réception active.

Aux termes évoqués plus haut d'aca-fan, de fan-scholar ou à l'inverse de scholar-fan, nous préférons le terme d' « ethno-fan $»^{34}$ qui renvoie au travail de l'ethnographe, immergé dans une communauté qu'il observe, tout en conservant l'expertise du fan et la connaissance de ses pratiques sociales, technologiques et médiatiques. Lorsque nous commençons une analyse de stratégies et dispositifs transmédiatiques autour de séries télévisées ou une étude des activités en ligne des fans, nous nous intégrons d'abord à la communauté en nous positionnant nous-même comme fan du programme, en participant aux débats et discussions, en live-tweetant les épisodes en utilisant les codes sémantiques des fans (hashtags propres, noms de communautés, noms de ships), en produisant des gif et des contenus sur Tumblr ${ }^{35}$. Ce travail, chronophage, prépare le terrain à l'analyse de la production mais également de la réception rendue possible par une bonne connaissance des pratiques des fans. De plus, cela nous donne une légitimité ${ }^{36}$ ainsi qu'une expertise, essentielle pour ensuite interroger les fans par le biais de questionnaires distribués en ligne sur les réseaux sociaux ou directement sur les forums. Ces questionnaires portent sur leurs pratiques et leur perception et réception des stratégies de production transmédiatiques.

L'ethno-fan développe une nouvelle démarche de recherche qui entraîne des méthodologies adaptées au contexte et à l'objet: immersion dans la communauté, recueil et aspiration des traces numériques et des travaux d'archivage des fans, navigation hypertexte. Une telle méthode est la condition pour que le chercheur possède une vision suffisamment complète du terrain et des pratiques lui permettant d'analyser un écosystème médiatique (production et réception) en pleine mutation et des narrations sérialisées multi-plateformes.

\section{NOTES}

1. Paul Booth, Lucy Bennett, Seeing Fans. Representations of fandom in media and popular culture, Londres, Bloomsbury, 2016.

2. Matt Hills, Fan cultures, Londres, Routledge, 2002. Toutes les traductions sont les nôtres.

3. Pierre Bourdieu, La Distinction. Critique sociale du jugement, Paris, Les Editions de Minuit, 1979.

4. Gabriel Segré, Fans de...Sociologie des nouveaux cultes contemporains, Paris, Armand Colin, 2014, p. 114.

5. Mélanie Bourdaa., Javier Lozano Delmar, « Case study of French and Spanish reception of Game of Thrones ", Transformative Works and Cultures, Special issue «Transnationalism, localization and 
translation in European Fandom (edited by Kustritz A.), vol. 19, 2015. http:// journal.transformativeworks.org/index.php/twc/article/view/608

6. Henry Jenkins, Textual Poacher. Television Fans and Participatory Culture, Londres, Routledge, 1992.

7. Paul Booth, Lucy Bennett, op. cit.

8. Nick Abercrombie, Brian Longhurst, "Fans and Enthusiasts", in The Fan Fiction Reader, University of Iowa Press, 2014. pp. 158-176.

9. John Fiske, «The Cultural Economy of Fandom ", in Lisa Lewis, The adoring audience: fan culture and popular media, Routledge, 1992. pp. 30-49.

10. Henry Jenkins, «La filk et la construction sociale de la communauté des fans de sciencefiction », in Hervé Glévarec, Éric Macé, Éric Maigret, Cultural Studies. Anthologie, Armand Colin / INA, Paris, 2008, pp. 212-222.

11. F. Coppa, "Fuck Yeah! Fandom is beautiful", Journal of fandom Studies, volume 2, numéro 1, 2014, pp. 73-82.

12. Ien Ang, Watching Dallas. Soap Opera and the melodramatic imagination, Londres, Routledge, 1985.

13. Sarah M. Corse, Jaime Hartless, "Sci-fi and skimpy outfits. Making boundaries and staking claims to Star Trek : into Darkness ", in Adrienne Trier-Bienick, Fan Girls and The Media. Creating Characters, Consuming Culture, New York, Rowman and Littlefield, 2015. pp. 1-20.

14. Paul Booth, Digital Fandom. New Media Studies, New York, Peter Lang, 2010. p. 10

15. Mark Duffet, Understanding Fandom: an Introduction to the Study of Media Fan Culture, New York, Bloomsbury, 2013. p. 21.

16. Patrice Flichy, Le Sacre de l'amateur. Sociologie des passions ordinaires à l'ère du numérique, Paris, Editions du Seuil et La République des Idées, 2010.

17. L. Allard, «Express Yourself 3.0 ! Le mobile comme média de la voix intérieure », in L. Allard, R. Odin, L. Creton (sous la dir. de), Téléphone mobile et création, Armand Colin, 2014.

18. Nancy Baym, Tune in, log on. Soaps, Fandom and Online Community, Londres, Sage Publications, 1999.

19. Christian Le Bart, Jean-Charles Ambroise, Les Fans des Beatles. Sociologie d'une passion, Rennes, Presses Universitaires de Rennes, 2000.

20. Adrienne Evans, Mafalda Stasi, «Desesperately seeking methodology : new directions in fan studies research », in Participations. Journal of Audience and Reception Studies, vol. 11, issue 2, 2014, pp. 4-23.

21. Mélanie Bourdaa (dir.), « les fan studies : enjeux et perspectives de la recherche sur les fans en France", in Revue Française des Sciences de l'Information et de la Communication, dossier «Emergences », numéro 7, 2015. https://rfsic.revues.org/1462 (consulté le 10 octobre 2017).

22. Booth P., "Augmenting fan/academic dialogue: new directions in fan studies ", Journal of Fandom Studies, vol. 1 n², 2013, p. 131.

23. Aurore Gallarino, "Jenkins Reloaded: évolution de la pensée d'Henry Jenkins de Textual Poachers à (l'avant) Spreadable Media », Terminal, $\mathrm{n}^{\circ}$ 112, 2015, p. 115-120.

24. Cécile Christofari, Matthieu J., Guitton, «L'aca-fan : aspects méthodologiques, éthiques et pratiques ", Revue Française des Sciences de l'Information et de la Communication, n 7, 2015. https:// rfsic.revues.org/1651\#tocto1n2

25. Paul Booth, ibid., p. 120.

26. Sam Ford, «Fan Studies : grappling with an 'undisciplined' discipline », Journal of Fandom Studies, Vol. $2 \mathrm{n}^{\circ} 1,2014$, pp. 53-71.

27. Stuart Hall, « Encoding and Decoding in the Television Discourse », conférence prononcée en 1973, accessible en ligne, https://core.ac.uk/download/pdf/81670115.pdf (26 janvier 2019).

28. David Morley, Family Television. Cultural Power and Domestic Leisure, Londres, Routledge, 1986.

29. Ien Ang, Watching Dallas. Soap Opera and the Melodramatic Imagination, Londres, Routledge, 1985. 
30. Janice Radway, Reading The Romance. Women, Patriarchy and Popular Literature, The University of North Carolina Press, 1984.

31. Paul Booth, 2013, op.cit.

32. Katie J. Ward, " Cyber-ethnography and the emergence of the virtually new community », in Journal of Information Technology, vol. 14, 2009. pp. 95-105.

33. Ulun Akturan, «A review of cyber ethnographic research : research technique to analyze virtual consumer communities », in Bogaziçi Journal, Vol. 23 n¹-2, 2009. pp. 1-18.

34. Je développe cette démarche dans Mélanie Bourdaa, «Le démarche "Ethno-fan»: De l'importance de l'immersion du chercheur dans les communautés de fans dans l'étude du Transmedia Storytelling", in Lécossais S., Quéméner N., En quête d'archives: bricolages méthodologiques en terrain médiatique, Ina, 2017 (à paraître).

35. Voir mon travail sur la communauté des fans de Battlestar Galactica dans Mélanie Bourdaa, "Taking a break from all your worries : Battlestar Galactica et les nouvelles pratiques télévisuelles des fans ", Questions de Communication, $n^{\circ} 22,2012$. p. 235-250.

36. Mélanie Bourdaa, op. cit., 2012.

\section{ABSTRACTS}

Les discours sur les publics fans sont pétris de fantasmes. Mais il est nécessaire d'étudier ces publics actifs, producteurs de sens et de contenus. Que sont les études de fans? Comment ontelles évolué ? Quelles sont les méthodologies pour analyser ces publics spécifiques ? Cet article se propose, à travers une revue de la littérature critique, de revenir à la fois sur les définitions du fan et de la fan culture et de comprendre comment les chercheurs peuvent appréhender ces publics.

\section{INDEX}

Mots-clés: fans, définition, méthodologie, fan culture, communautés

\section{AUTHOR}

\section{MÉLANIE BOURDAA}

MICA, Université Bordeaux Montaigne 